EGU2020-8071

EGU General Assembly 2020

(c) Author(s) 2020. This work is distributed under

the Creative Commons Attribution 4.0 License.

\title{
SKS splitting observations across the Iranian plateau and Zagros: the role of lithosphere deformation and mantle flow
}

\author{
Ayoub Kaviani ${ }^{1}$, Meysam Mahmoodabadi ${ }^{1,2}$, Georg Rümpker ${ }^{1}$, Farzam Yamini-Fard ${ }^{2}$, Mohammad \\ Tatar $^{2}$, Ali Moradi ${ }^{3}$, and Faramarz Nilfouroushan ${ }^{4,5}$ \\ ${ }^{1}$ Johann Wolfgang Goethe University, Frankfurt, Geowissenschaften, Geophysics, Frankfurt am Main, Germany \\ (kaviani@geophysik.uni-frankfurt.de) \\ ${ }^{2}$ International Institute of Earthquake Engineering and Seismology, Tehran, Iran \\ ${ }^{3}$ Institute of Geophysics, University of Tehran, Tehran, Iran \\ ${ }^{4}$ Faculty of Engineering and Sustainable Development (ATM) University of Gävle, Sweden \\ ${ }^{5}$ Department of Geodetic Infrastructure, Lantmäteriet, Gävle, Sweden
}

We used more than one decade of core-refracted teleseismic shear (SKS) waveforms recorded at more than 160 broadband seismic stations across the Iranian plateau and Zagros to investigate seismic anisotropy beneath the region. Splitting analysis of SKS waveforms provides two main parameters, i.e., fast polarization direction and split delay time, which serve as proxies for the trend and strength of seismic anisotropy beneath the stations. Our observation revealed a complex pattern of splitting parameters with variations in the trend and strength of anisotropy across the tectonic boundaries. We also verified the presence of multiple layers of anisotropy in conjunction with the lithosphere deformation and mantle flow field. Our observation and modeling imply that a combined system of lithosphere deformation and asthenospheric flow is likely responsible for the observed pattern of anisotropy across the Iranian Plateau and Zagros. The rotational pattern of the fast polarization directions observed locally in Central Zagros may indicate the diversion of mantle flow around a continental keel beneath the Zagros. The correlation between the variation in lithosphere thickness and the trend of anisotropy in the study area implies that the topography of the base of lithosphere is also a determining factor for the pattern of mantle flow inferred from the observations. 